\title{
FT-IR/PAS Studies of Cu(II)-EDTA Complexes Sorption on the Chelating Ion Exchangers
}

\author{
D. KoŁodyńska, Z. Hubicki and S. Pasieczna-Patkowska \\ Faculty of Chemistry, University of Maria Curie-Skłodowska \\ pl. M. Curie-Skłodowskiej 2, 20-031 Lublin, Poland
}

\begin{abstract}
The possibility of removal of copper(II) complexes with ethylenediaminetetraacetic acid (EDTA) from waters and wastewaters by the chelating ion exchangers Lewatit TP 214, Lewatit TP 260 and Diaion CR-20 as well as the mechanism of their sorption onto them were investigated. In order to describe the sorption process of $\mathrm{Cu}(\mathrm{II})$ ions in the presence of EDTA, the FT-IR/PA spectra of the abovementioned chelating ion exchangers were recorded.
\end{abstract}

PACS numbers: $82.80 . \mathrm{Kq}, 81.70 . \mathrm{Cv}, 82.50 .-\mathrm{m}$

\section{Introduction}

Removal of high concentrations of heavy metals is normally achieved by technologies such as precipitation, coagulation/flocculation, sedimentation and filtration. In many situations these processes are inefficient or do not reduce metal concentrations below the accepted standards. Additionally, heavy metal ions are often present in various industrial wastewaters together with complexing agents. There is an increasing concern about the direct or indirect potential effects of the presence of such chelators because of the possibility of mobilization of contaminating metal ions. The presence of EDTA may prevent from precipitation of heavy metal ions in solution or, on the contrary, cause a dissolution effect of heavy metals adsorbed in sediments. EDTA can also solubilize radioactive metals and increase their environmental mobility. Contribution of EDTA to the eutrophication water processes is also possible. Therefore the most important technologies for the removal of toxic metals or reduction of their concentrations are adsorption, ion exchange, solvent extraction, phytoextraction and biosorption. Anionic complexes of heavy metal ions with complexing agents can be eliminated by means of anion exchangers. Recent studies have also shown that chelating resins could be used for selective removal and recovery of metals in the presence of strong organic ligands [1-4]. Therefore, the aim of this paper was to examine the removal of $\mathrm{Cu}$ (II) ions in the presence of EDTA on the chelating ion exchangers of different types.

\section{Experimental}

\subsection{Anion exchangers}

The following chelating resins were selected for the study: Lewatit TP 214 with thiouronium functional, its total ion exchange capacity is $2.0 \mathrm{meq} / \mathrm{mL}$, the particle size of $>0.55 \mathrm{~mm}$; Lewatit TP 260 with aminomethylphosphonate functional groups, its total ion exchange capacity is $2.4 \mathrm{meq} / \mathrm{mL}$, and the particle size of $>0.63 \mathrm{~mm}$ as well as Diaion CR-20 with polyamine functional groups, its total ion exchange capacity is about $0.80 \mathrm{meq} / \mathrm{mL}$ and the particle size of $0.3-0.33 \mathrm{~mm}$. Prior to the use they were washed with $1 \mathrm{M} \mathrm{NaOH}, 1 \mathrm{M} \mathrm{HCl}$ and deionized water.

\subsection{Solutions of $C u(I I)$ complexes with EDTA}

The initial aqueous solutions of copper(II) complexes with EDTA were prepared by dissolving the equimolar amount of $\mathrm{Cu}(\mathrm{II})$ chloride in the EDTA solution. The initial $\mathrm{pH}$ for the studied complexes was 3.5. In this condition copper(II) tends to form thermodynamically stable complexes, so the following equations can be assumed: $\mathrm{M}^{2+}+\mathrm{H}_{2}$ edta $\rightleftharpoons[\mathrm{M}($ edta $)]$ and $\mathrm{M}^{2+}+$ Hedta $^{3-} \rightleftharpoons$ $[\mathrm{M}(\text { edta })]^{-}$.

\subsection{The static method}

The batch experiments were conducted according to the following procedure: about $0.5 \mathrm{~g}$ of appropriate dry ion exchanger was placed in a $100 \mathrm{~cm}^{3}$ stoppered conical flask containing $50 \mathrm{~cm}^{3}$ of $\mathrm{Cu}$ (II) complexes of an initial concentration $c_{0}$. The flask was shaken using the laboratory shaker of ELPHINE type 357 produced in Poland. The measurements were made at the constant temperature in the two parallel series. In order to determine the content of $\mathrm{Cu}$ (II) in the raffinate the atomic absorption spectroscopy (AAS) method was used. The amount of metal sorbed per unit mass of ion exchanger at the specific time and at the equilibrium $\left(q_{\mathrm{t}}, q_{\mathrm{e}}\right)$ was calculated from the equations 


$$
\begin{aligned}
& q_{\mathrm{t}}=\frac{\left(c_{0}-c_{\mathrm{t}}\right) V}{m}, \\
& q_{\mathrm{e}}=\frac{\left(c_{0}-c_{\mathrm{e}}\right) V}{m},
\end{aligned}
$$

where $c_{0}$ - initial concentration of $\mathrm{Cu}$ (II) in the aqueous phase $(\mathrm{mg} / \mathrm{L}), c_{\mathrm{t}}$ - concentration of $\mathrm{Cu}(\mathrm{II})$ in the aqueous phase at time $t(\mathrm{mg} / \mathrm{L}), c_{\mathrm{e}}$ - concentration of $\mathrm{Cu}(\mathrm{II})$ in the aqueous phase at equilibrium $(\mathrm{mg} / \mathrm{L}), V$ - volume of the solution $(L), m$ - mass of the ion exchanger $(\mathrm{g})$.

\subsection{Spectroscopic characteristics}

The Fourier transform infrared photoacoustic spectroscopy (FT-IR/PA) spectra were recorded by means of a Bio-Rad Excalibur 3000MX spectrometer equipped with the photoacoustic detector MTEC 300 (in the He atmosphere in a detector) over the $4000-400 \mathrm{~cm}^{-1}$ range at the resolution of $4 \mathrm{~cm}^{-1}$. The spectra were normalized with reference to the MTEC carbon black standard. The interferograms of 1024 scans were averaged for each spectrum.

\section{Results and discussion}

As follows from the obtained results for chelating ion exchangers the amount of $\mathrm{Cu}(\mathrm{II})$ complexes increases with time and remains constant after the contact time of about $60 \mathrm{~min}$. The time profile of copper(II) complexes uptake is a single, smooth and continuous curve leading to saturation, suggesting the possible monolayer coverage of $\mathrm{Cu}(\mathrm{II})$ on the surface of ion exchanger. A series of contact time experiments undertaken at various $\mathrm{pH}$ values shows maximum removal in the $\mathrm{pH}$ range $2-4$. In this range the sorption is very fast and then gradually decreases. At $\mathrm{pH}>8$ the sorption efficiency increases due to precipitation of hydroxocomplexes. The exemplary results obtained for Lewatit TP 260 are presented in Fig. 1.

In order to describe the sorption mechanism of copper(II) in the presence of EDTA, the photoacoustic method was used.

The recordered FT-IR/PA spectra for Lewatit TP 260 and Lewatit TP 214 are presented in Fig. 2.

It was noticed that for chelating ion exchangers in the higher frequency region at $3400 \mathrm{~cm}^{-1}$ very broad and strong bands of $\mathrm{O}-\mathrm{H}$ as well as $\mathrm{N}-\mathrm{H}$ stretching were observed. The presence of water in the ion exchanger phase was also found at $1636 \mathrm{~cm}^{-1}(\delta(\mathrm{O}-\mathrm{H}))$.

On the lower frequency side of this band, the peaks related to the asymmetric stretching bonds $\nu_{\text {as }}(\mathrm{C}-\mathrm{H})$ of the ring $\mathrm{C}-\mathrm{H}$ at $3023 \mathrm{~cm}^{-1}$ and the asymmetric stretching bonds $\nu_{\text {as }}\left(-\mathrm{CH}_{2}\right)$ of $-\mathrm{CH}_{2}$ groups at $2926 \mathrm{~cm}^{-1}$ and symmetric stretching bonds $\nu_{\mathrm{s}}\left(-\mathrm{CH}_{2}\right)$ at $2856 \mathrm{~cm}^{-1}$ of the cross-linked polystyrene were observed, respectively. The ring carbon-carbon stretching $\nu_{\text {as }}(\mathrm{C}=\mathrm{C})$ bonds occur at $1508,1489 \mathrm{~cm}^{-1}$ and the scissoring vibrations of the methylene groups $\left(\delta_{\text {as }}\left(-\mathrm{CH}_{2}\right)\right)$ occur at 1485,1455 and $1417 \mathrm{~cm}^{-1}$ as well as at $1393 \mathrm{~cm}^{-1}$. The peaks at
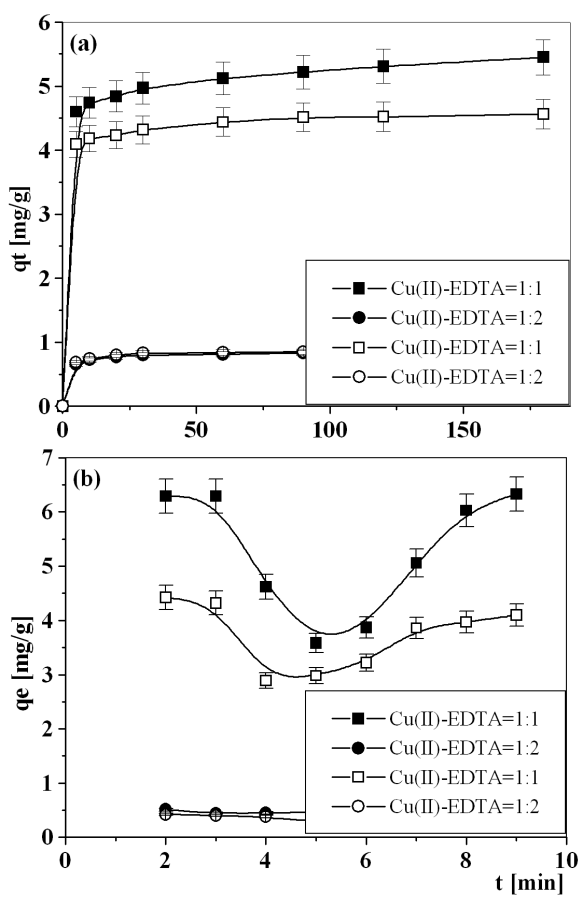

Fig. 1. Effect of the phase contact time (a) and pH value (b) on the sorption of $\mathrm{Cu}$ (II) complexes with EDTA in the 1:1 and 1:2 systems on Lewatit TP 260 in the $\mathrm{Na}^{+}$(closed points) and $\mathrm{H}^{+}$(opened points) forms.

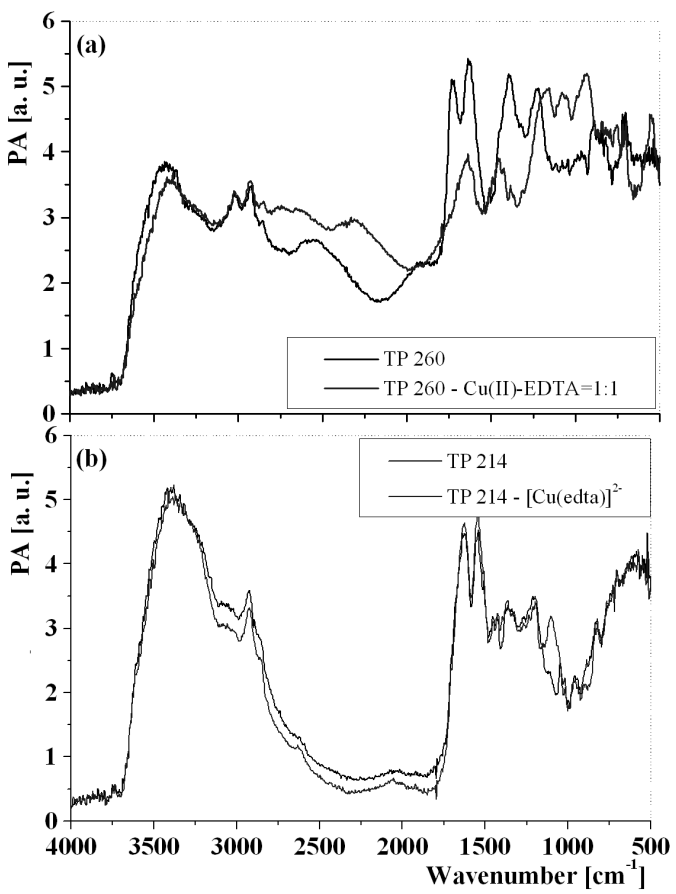

Fig. 2. FT-IR/PA spectra of Lewatit TP 260 (a) and Lewatit TP 214 (b) before and after the sorption of $\mathrm{Cu}$ (II) complexes with EDTA. 
974 and $702 \mathrm{~cm}^{-1}$ can be ascribed to the deformation vibrations of 1,4-disubstituted benzene ring (ST-DVB). In the spectra of Lewatit TP 260 and Lewatit TP 214 the deformation vibrations of 1,3-disubstituted benzene ring (ST-DVB) are also visible at 826 and $759 \mathrm{~cm}^{-1}[5,6]$.

For Lewatit TP 260 with aminomethylphosphonate functional groups $\left(-\mathrm{CH}_{2} \mathrm{NHCH}_{2} \mathrm{PO}_{3} \mathrm{H}_{2}\right)$ (Fig. 2a), the ring carbon-carbon stretching and the scissoring vibrations of the methylene groups are weaker than for Lewatit TP 214. The strong bands related to the amine group can be assigned to the peaks at $3420 \mathrm{~cm}^{-1}$. The $\mathrm{C}-\mathrm{N}$ absorption is found in the range from 1200 to $1350 \mathrm{~cm}^{-1}$. The compounds with the $\mathrm{P}-\mathrm{OH}$ group have two broad bands of medium intensity at 2732 and $2323 \mathrm{~cm}^{-1}$, which are due to the $\mathrm{O}-\mathrm{H}$ stretching vibrations and a strong band due to the $\mathrm{P}-\mathrm{O}$ stretching vibration at 1393 and $1220 \mathrm{~cm}^{-1}$. The bands at 1631 and $1723 \mathrm{~cm}^{-1}$ are related to the deformation band of $-\mathrm{NH}$ group. As follows from the literature data [7-10] the aminomethylphosphonate groups are potentially tridentate ligands, having two bonding sites at a phosphonic group and one coordination site at the secondary nitrogen atom. The sorption of heavy metal ions can proceed by their coordination interaction and then through their ionic interaction. The ion exchangers containing such groups are characterized by large selectivity towards heavy metal ions and their sorptive properties are determined by the extent of phosphonic group ionization. This is due to the electronacceptor effect of the cationic centre $\equiv \mathrm{N}$ or $\equiv \mathrm{P}=$ through the alkyl fragment. After the sorption of $\mathrm{Cu}(\mathrm{II})$ ions in the presence of EDTA there appears a band connected with asymmetric stretching vibrations of the $\mathrm{C}-\mathrm{O}$ band at $1641 \mathrm{~cm}^{-1}$ and symmetric stretching vibrations at $1453 \mathrm{~cm}^{-1}$. However, the band connected with the vibrations of the $-\mathrm{NH}$ group disappears (Fig. 2a) which can indicate decomposition of the $\mathrm{Cu}(\mathrm{II})$-EDTA complex and formation of the coordination bond between a nitrogen atom and a phosphorus atom. The most favourable mode of chelation when $\mathrm{pH}$ increases towards the weak acid range is formation of the following 4-membered ring:

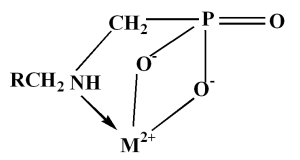

In the case of Lewatit TP 214 with the thiouronium functional groups containing sulphide and nitrogen atoms, the board peak at the higher wave numbers $3200-3400 \mathrm{~cm}^{-1}$ should be associated with the asymmetric and symmetric stretching bonds of the $-\mathrm{NH}_{2}$ groups of thiouronium (Fig. 2b). As follows from the literature data [11], the thiouronium functional groups of the studied ion exchanger are potentially to form coordinate bonds through both sulphur and nitrogen atoms even though the extremely low basicity of the ligand prevents from the formation of nitrogen-metal bonds. In an acidic medium the thiouronium group is protonated and the proton will be bonded to the sulphide atom favouring the bonding of the metal ions to nitrogen atoms, so the
$\mathrm{pH}$ value is the fundamental parameter for the formation of metal-thiouronium complexes. The possible forms of this group can be as follows:

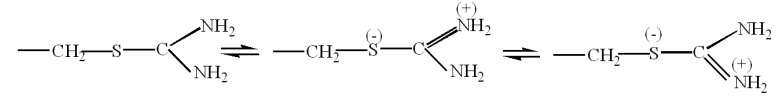

The bands related to the functional group of Lewatit TP 214 are also present at $519 \mathrm{~cm}^{-1}$ (skeletal deformation $\delta(\mathrm{C}-\mathrm{S})$ and stretching bands $\nu(\mathrm{C}-\mathrm{S}))$, at $1103 \mathrm{~cm}^{-1}$ it is related to the deformation band of $-\mathrm{NH}_{2}$ groups $\left(\delta\left(-\mathrm{NH}_{2}\right)\right)$ and the peak at $1394 \mathrm{~cm}^{-1}$ to the combined $\mathrm{C}-\mathrm{N}$ and $\mathrm{C}-\mathrm{S}$ bond $(\nu(\mathrm{C}-\mathrm{N}) /(\mathrm{C}-\mathrm{S}))$.

After the sorption of $\mathrm{Cu}(\mathrm{II})$ ions in the presence of EDTA, the FT-IR/PA spectrum of Lewatit TP 214 possesses two strong bands at $1629 \mathrm{~cm}^{-1}$ and $1542 \mathrm{~cm}^{-1}$ assigned to the asymmetrical and symmetrical stretching of the $\mathrm{COO}^{-}$group, respectively $\left(\nu_{\text {as }}\left(\mathrm{COO}^{-}\right)\right.$and $\left.\nu_{\mathrm{S}}\left(\mathrm{COO}^{-}\right)\right)$. This can be also related to the deformation band of $-\mathrm{NH}_{2}$ group. Other peaks for the $-\mathrm{COO}^{-}$group are at $1424-1455 \mathrm{~cm}^{-1}$ and at $1365-1395 \mathrm{~cm}^{-1}$ as well as two additional peaks at $900-960$ and $815-865 \mathrm{~cm}^{-1}$.

In the obtained spectrum the band at $1607 \mathrm{~cm}^{-1}$ assigned to the $[\mathrm{Cu}(\text { edta })]^{2-}$ complex is difficult to identify because of ion exchanger band overlapping. In the case of $\mathrm{H}_{4}$ edta (protonated form of EDTA) carbonyl absorption occurs near $1700 \mathrm{~cm}^{-1}$ but in the case of metalEDTA complexes absorption maxima are found in the range $1590-1650 \mathrm{~cm}^{-1}[5,6]$. The position of the carbonyl band in the infrared spectra of EDTA complexes was employed as the indication of the nature of $\mathrm{M}-\mathrm{O}$ bond. It was documented that for the $q / r$ ratio ( $q$ is the charge of metal ion and $r$ its ionic radius) larger than 3.6 the carbonyl absorption maxima occur in the 1625 to $1650 \mathrm{~cm}^{-1}$ region indicating the covalent bonding but for $q / r$ less than 3.6 they are in the 1590 to $1615 \mathrm{~cm}^{-1}$ region and indicate that the bonding is primarily ionic. When the peaks occur between these regions the bonding is probably partially ionic and partially covalent [12].

In the case of Diaion CR-20 the FT-IR/PA spectra of the ion exchanger before and after sorption of $\mathrm{Cu}$ (II) complexes with EDTA are very similar, therefore they are not presented.

\section{Conclusions}

The FT-IR/PAS method is useful to explain the nature of the $\mathrm{M}-\mathrm{O}$ bond and to describe the mechanism of sorption process of $\mathrm{Cu}(\mathrm{II})$ ions in the presence of the complexing agent EDTA. The obtained results indicate possible application of the chelating ion exchangers Lewatit TP 260 and Lewatit TP 214 in technologies of $\mathrm{Cu}$ (II) removal from aqueous solutions especially wastewater streams containing strong organic ligands.

\section{References}

[1] C. Oviedo, J. Rodríguez, Quimica Nova 26, 901 (2003). 
[2] E. Pehlivan, T. Altun, J. Hazard, Materials 140, 299 (2007).

[3] R. Raghavan, C.V. Bhatt, Hydrometallurgy 50, 169 (1998).

[4] T.A. Kurniawan, G.Y.S. Chan, W.H. Lo, S. Babel, Chem. Eng. J. 118, 83 (2006).

[5] G. Socrates, Infrared and Raman Characteristic Group Frequencies. Tables and Charts, Wiley, Chichester 2001.

[6] K. Nakamoto, Infrared and Raman Spectra of Inorganic and Coordination Compounds, Wiley, New York 1986.

[7] S.K. Sahni, R. Bennekom, J. Reedijk, Polyhedron 4, 164 (1985).
[8] S.V. Kertman, G.M. Kertman, Y.A. Leykin, Thermochim. Acta 256, 227 (1995).

[9] J. Mazur, B. Pustelny, Mol. Quant. Acoust. 28, 195 (2007).

[10] T. Starecki, Acta Phys. Pol. A 114, A-199 (2008).

[11] A. Gherrou, H. Kerdjoudj, R. Molinari, E. Drioli, Sep. Sci. Technol. 28, 235 (2002).

[12] D.T. Sawyer, P.J. Paulsen, J. Am. Chem. Soc. 80, 1597 (1958). 